Published in final edited form as:

Gynecol Oncol. 2009 December ; 115(3): 460-465. doi:10.1016/j.ygyno.2009.09.011.

\title{
Sunitinib Malate in the treatment of recurrent or persistent uterine leiomyosarcoma: A Gynecologic Oncology Group phase II study
}

\author{
Martee L. Hensley, MD ${ }^{1}$, Michael W. Sill, PhD ${ }^{2,3}$, Dennis R. Scribner Jr., MD ${ }^{4}$, Jubilee Brown, \\ $M^{5}$, Robert L. DeBernardo, MD 6 , Ellen M. Hartenbach, MD ${ }^{7}$, Carolyn K. McCourt, MD ${ }^{8}$, \\ James R. Bosscher, MD $^{9}$, and Paola A. Gehrig, MD ${ }^{10}$ \\ ${ }^{1}$ Memorial Sloan-Kettering Cancer Center, New York, NY \\ ${ }^{2}$ GOG Statistical and Data Center, Roswell Park Cancer Institute, Buffalo, NY \\ ${ }^{3}$ Dept. of Biostatistics, University at Buffalo, Buffalo, NY \\ ${ }^{4}$ Carilion Clinic, Roanoke, VA 24014 \\ ${ }^{5}$ The University of Texas, M.D. Anderson Cancer Center, Houston, TX 77230 \\ 6University Hospitals Case Medical Center, Cleveland, $\mathrm{OH} 44106$ \\ ${ }^{7}$ University of Wisconsin Hospital - Madison, Madison, WI \\ ${ }^{8}$ Women \& Infants Hospital, Providence, RI 02905 \\ ${ }^{9}$ St. John's Health System, Springfield, MO 65804 \\ 10University of North Carolina at Chapel Hill, Chapel Hill, NC 27599
}

\section{Abstract}

\begin{abstract}
Purpose-New agents are needed for patients with metastatic uterine leiomyosarcoma who progress after treatment with doxorubicin or gemcitabine-docetaxel. Agents targeting tumor vasculature have potential for activity in leiomyosarcoma. We aimed to assess the activity of sunitinib in patients with recurrent uterine leiomyosarcoma who had received one or two prior therapies by determining the frequency of patients who survived progression-free for at least six months or who achieved objective tumor response. We also aimed to characterize the toxicity of sunitinib and to estimate time-to-progression.
\end{abstract}

\begin{abstract}
Patients and Methods-Eligible patients with uterine leiomyosarcoma were treated with sunitinib $50 \mathrm{mg}$ by mouth daily for four weeks, with two weeks rest. Tumor response and progressionfree status were assessed every six weeks.
\end{abstract}

Results-Twenty-three of 25 patients enrolled were evaluable for efficacy (two wrong histologies). The median number of cycles was one. Two of 23 patients achieved a partial response $(8.7 \%, 90 \%$ two-sided, binomial confidence interval (CI) $1.6-24.9 \%$ ). Four patients remained progression-free

() 2009 Elsevier Inc. All rights reserved.

Corresponding Author: Martee L. Hensley, MD, Memorial Sloan-Kettering Cancer Center, Department of Medical Oncology, 1275 York Avenue, New York, NY 10065, Telephone: 212-639-6902, FAX: 212-717-3272, hensleym@ mskcc.org.

Publisher's Disclaimer: This is a PDF file of an unedited manuscript that has been accepted for publication. As a service to our customers we are providing this early version of the manuscript. The manuscript will undergo copyediting, typesetting, and review of the resulting proof before it is published in its final citable form. Please note that during the production process errors may be discovered which could affect the content, and all legal disclaimers that apply to the journal pertain.

CONFLICT OF INTEREST STATEMENT

None of the authors have any conflict of interest to declare. 
at six months $(17.4 \%, 90 \%$ two-sided, binomial confidence interval 6.2-35.5\%). Toxicities included: grade 3 neutropenia (17.4\%); grade 3 thrombocytopenia (13\%); grade 3 anemia (17.4\%); grade 3-4 lymphopenia (8.7\%); grade 3-4 fatigue (30\%); grade 3 vomiting/diarrhea (21.7\%); skin rash/handfoot syndrome, grade $2(13 \%)$, grade $3(4.3 \%)$; hypertension, grade $2(39 \%)$, grade $3(4.3 \%)$; grade 2 decrease in cardiac ejection fraction (4.3\%), and grade 3 thrombosis (4.3\%). Median progressionfree survival was 1.5 months.

Conclusion-Sunitinib fails to achieve sufficient objective response or sustained disease stabilization as second- or third-line treatment for uterine leiomyosarcoma.

\section{Keywords}

uterine leiomyosarcoma; sunitinib

\section{INTRODUCTION}

Uterine leiomyosarcoma (LMS) is a high-risk malignancy that is diagnosed in only a few thousand women each year in the United States. ${ }^{1}$ Median survival for women with advanced or unresectable, recurrent disease is less than one year. ${ }^{2}$ Treatment regimens that can achieve objective response in patients with advanced or recurrent uterine LMS include doxorubicin with or without ifosfamide (response rates 25-30\%) and fixed dose-rate gemcitabine plus docetaxel (response rate $35.8 \%$ as first-line treatment; $27 \%$ as second-line treatment). ${ }^{3-6}$ Single agents with moderate activity in LMS include ifosfamide (response rate 17.2\%) and gemcitabine (bolus infusion achieved a $20 \%$ response rate among women with uterine LMS who had received $0-1$ prior cytotoxic regimen). ${ }^{7,8}$ In soft tissue sarcoma, trabectedin achieved a response rate of $8 \%$ among patients with prior treatment and $17 \%$ as first-line therapy. ${ }^{9}, 10$ Trabectedin is currently being studied as first-line treatment for advanced uterine LMS in a Gynecologic Oncology Group (GOG) phase II study. Phase II trials have shown the following agents to be inactive: cisplatin, mitoxantrone, amonifide, oral etoposide, diazoquone (AZQ), intravenous etoposide, topotecan, paclitaxel, thalidomide, and trimetrexate. ${ }^{11-21}$

Novel agents are needed for uterine LMS as no treatment regimen is curative. Interruption of the vascular endothelial growth factor (VEGF) pathway is a novel approach to cancer therapy that may reduce tumor vascularity and thus limit tumor growth. VEGF and/or VEGF receptors are expressed in a variety of tumor types, including gynecologic cancers, with higher levels of vascularity associated with poorer prognosis. ${ }^{22-25}$ VEGF-trap (aflibercept) has been shown to inhibit tumor growth in mouse xenograft models, including a rhabdomyosarcoma model. ${ }^{26} \mathrm{In}$ a phase I study of single-agent, intravenous aflibercept, one heavily-pretreated patient with metastatic uterine LMS achieved a minor response. ${ }^{27}$

Sunitinib is a novel, multi-targeted, small molecule in hibitor of the receptor tyrosine kinases (RTKs) involved in tumor proliferation and angiogenesis, including vascular endothelial growth factor receptor-1 (VEGFR-1), -2, and -3, platelet-derived growth factor receptor (PDGFR) $-\alpha$ and $-\beta$, stem cell factor receptor (KIT), the tyrosine kinase (TK) receptor encoded by the ret proto-oncogene, and fms-like tyrosine kinase 3 (Flt3) ${ }^{28}$ Chronic oral dosing with sunitinib is expected to inhibit PDGF- and VEGF-driven angiogenesis and as a consequence, limit solid tumor growth. Because angiogenesis is necessary for the growth and metastasis of solid tumors, and VEGF is believed to have a pivotal role in this process, sunitinib treatment may have clinical activity in uterine LMS.

The Gynecologic Oncology Group (GOG) conducted this phase II trial of sunitinib to determine the activity of sunitinib as second-line or third-line therapy among women with advanced or recurrent uterine LMS. Because a tumor vasculature-targeted agent was expected to preserve disease stability more than achieve objective response, the trial was designed to consider 
progression-free status of patients at six months in addition to objective tumor response for evaluation of efficacy.

\section{MATERIALS AND METHODS}

\section{Patients}

Women with persistent or recurrent uterine leiomyosarcoma after treatment with one or two prior cytotoxic regimens, and who had measurable disease that was not considered curable were eligible for this study. Histologic confirmation was required and accomplished by central review of the GOG Pathology Committee. Prior hormonal therapy was permitted, but prior anti-angiogenic/non-cytotoxic treatment was not. Patients were permitted to have had prior pelvic radiotherapy for uterine LMS. Prior surgery, chemotherapy and/or radiation must have been completed at least four weeks prior to enrollment.

Patients were required to have GOG performance status of $0-2$, and adequate bone marrow function (absolute neutrophil count (ANC) greater than or equal to 1,500/microliter, platelets greater than or equal to $100,000 /$ microliter, hemoglobin greater than or equal to $9 \mathrm{gm} / \mathrm{dl}$ ); renal function (creatinine less than or equal to 1.5 xinstitutional upper limit of normal); hepatic function (bilirubin less than or equal to 1.5 xinstitutional upper limit of normal, and serum glutamic oxaloacetic transaminase (SGOT) and alkaline phosphatase less than or equal to 2.5 $x$ institutional upper limit of normal); and neurologic function (baseline neuropathy (sensory and motor) less than or equal to Common Toxicity Criteria grade 1).

Patients were required to have adequate cardiac function (baseline electrocardiogram with QTc $<500$ milliseconds and without evidence of serious ventricular arrhythmia, and measured cardiac ejection fraction within the institutional range of normal) and adequate blood pressure control (systolic blood pressure less than $140 \mathrm{mmHg}$, and diastolic blood pressure less than 90 $\mathrm{mmHg}$ ). Patients requiring therapeutic doses of warfarin were not eligible; however treatment with therapeutic doses of low molecular weight heparin was permitted, provided the international normalized ratio was less than or equal to 1.5. Patients unable to swallow and absorb tablets were not eligible. Patients with a serious or non-healing wound, ulcer, bone fracture, history of abdominal fistula, gastrointestinal perforation, or intra-abdominal abscess within 28 days of treatment, any history of cerebrovascular accident (CVA) or transient ischemic attack within 12 months prior to study entry, history of myocardial infarction, cardiac arrhythmia, stable/unstable angina, symptomatic congestive heart failure, or coronary/ peripheral artery bypass graft or stenting within 12 months prior to study entry, history of pulmonary embolism within the past 12 months, or Class III or IV heart failure as defined by the New York Heart Association (NYHA) functional classification system were not eligible. Concomitant use of the potent inducers or inhibitors of CYP3A4 (for example: amiodarone, isoniazid, aminoglutethimide) was not permitted.

All patients signed written, informed consent. The protocol and consent were reviewed and approved annually by participating institutions' Institutional Review Boards.

\section{Treatment plan}

All participants had baseline imaging (magnetic resonance imaging (MRI), or computed tomography (CT) scan of chest, abdomen, and pelvis) within four weeks of starting therapy, which was repeated following every six-week cycle of treatment to assess response. History and physical examination, and assessment of toxicities were done each cycle. Complete blood counts were monitored weekly and comprehensive metabolic panels on day one of each cycle. Patients with NYHA class II cardiac dysfunction, a history of previous anthracycline treatment, or a history of thoracic radiation had repeat evaluations of left ventricular ejection fraction 
every other cycle. Toxicities were graded according to National Cancer Institution Common Toxicity Criteria version 3.0 (CTC 3.0).

Sunitinib, $50 \mathrm{mg}$ by mouth, was given daily in the morning with or without food for four weeks, followed by a two-week no-treatment rest. Six weeks was termed one cycle of treatment. Patients completed a medication and blood pressure monitoring diary.

Treatment continued until time of objective pr ogression of disease, or unacceptable toxicity.

Patients received day one treatment of each cycle provided the ANC was greater than or equal to $1500 /$ microliter and platelet count greater than or equal to $100,000 /$ microliter.

Sunitinib was held for grade 3 or 4 neutropenia or thrombocytopenia until recovery to grade 2 or less. Grade 4 neutropenia or thrombocytopenia required a dose reduction to $37.5 \mathrm{mg}$ daily. Sunitinib was also held for: grade 2 or worse neuropathy or renal toxicity; grade 3 or worse hepatic toxicity, or fever/chills/influenza-like symptoms, or fatigue/asthenia, or hand-foot syndrome skin toxicity. Sunitinib was held for QTc prolongation to greater than or equal to 550 milliseconds. Re-treatment with a reduced dose of sunitinib was generally permitted if toxicity improved. Patients who developed systolic blood pressure $\geq 140 \mathrm{mmHg}$ or diastolic blood pressure $\geq 90 \mathrm{mmHg}$ were treated with antihypertensive medications and monitored. If grade 3 or 4 hypertension developed, sunitinib was held. Patients with grade 4 hypertension were removed from study treatment. Others could be re-treated with a reduced dose once blood pressure control was achieved. Patients with a decrease in the left ventricular ejection fraction to below institutional normal limits had a repeat assessment after one to two weeks. If the decreased ejection fraction was confirmed, sunitinib was discontinued. If the ejection fraction recovered within three weeks, re-treatment with sunitinib was permitted at a reduced dose and with careful monitoring of the ejection fraction.

Objective response and disease progression were assessed by definitions in Response Evaluation Criteria In Solid Tumors (RECIST). Complete response (CR) is disappearance of all target and non-target lesions and no evidence of new lesions documented by two disease assessments at least four weeks apart. Partial response (PR) is at least a 30\% decrease in the sum of longest dimensions (LD) of all measurable target lesions taking as reference the baseline sum of $L D$. There can be no unequivocal progression of non-target lesions and no new lesions. Documentation by two disease assessments at least four weeks apart is required. In the case where the ONLY target lesion is a solitary pelvic mass measured by physical exam, which is not radiographically measurable, a $50 \%$ decrease in the LD is required. Progression of disease requires at least a $20 \%$ increase in the sum of $\mathrm{LD}$ of target lesions taking as references the smallest sum LD or the appearance of new lesions or death due to disease or global deterioration due to disease. Stable disease (SD) is any condition not meeting the above criteria.

\section{Statistical design}

The primary objective of this study was to assess the efficacy and toxicity of sunitinib in patients with recurrent or persistent LMS of the uterus. The primary endpoints of the study were the frequency of patients who survived progression-free for at least six months and the frequency of patients who had an objective tumor response (partial or complete). Given the importance of drug induced toxicities, the frequency and severity of adverse events as assessed by Common Toxicity Criteria Adverse Event v3.0 was also considered a primary endpoint. The secondary endpoints were the duration of progression-free survival (PFS) and overall survival (OS). Time at risk was measured from the date of registration. The endpoint for PFS was disease progression or death. The endpoint for OS was death by any cause. 
The study employed a two-stage group sequential design with an early stopping rule in the event that the treatment demonstrated insufficient activity. ${ }^{29}$ The targeted accrual for the first stage was 19 eligible and evaluable patients but was allowed to deviate for administrative purposes. If at least three patients had responses or at least five patients were observed to survive progression-free for six months, a second stage of accrual was to be initiated with a cumulative targeted sample size of 42 patients. If more than seven patients had responses or more than 10 patients were observed to survive progression-free for six months, then the study regimen would be deemed clinically interesting and worthy of further investigation in a phase III trial. The specific set of critical values depended on the actual accrual to each stage.

A historical dataset from prior GOG phase II studies in uterine leiomyosarcoma was used to establish the proportion of patients with objective responses or with progression-free survival of 6 months or more that were considered not clinically interesting. ${ }^{8}, 14,18,20,21$ Based on an analysis of these cohorts, it was determined that agents capable of truly achieving no more than $10 \%$ objective responses and no more than $15 \%$ progression-free survival at six months were to be classified as not clinically interesting. In contrast, regimens capable of yielding a true proportion of $30 \%$ of patients with objective responses or 35\% PFS at six months would be considered clinically interesting and should be considered for further assessment in a phase III study.

If the targeted accrual was attained, then the study had at least a $48 \%$ probability of terminating early when the agent is uninteresting. If the study continued into the second stage, the probability of the study declaring an inactive agent as being interesting was not greater then $8.4 \%$. The power of the study at detecting a truly active regimen (by either response or PFS for six months) was at least $89 \% .^{29}$

The CONSORT diagram is provided in Figure 1.

\section{RESULTS}

\section{Patient characteristics}

Twenty-five women were enrolled on study through ten participating GOG institutions. The first stage of accrual was completed within eight months. Twenty-three women were evaluable for efficacy and toxicity (two patients had wrong histology on central review). The median age was 56 (range $34-75$ ). All but one patient had a GOG performance status of $0-1$. Eighteen (78.3\%) were white; four (17.4\%) African American; one (4.3\%) American Indian. Nine patients $(39.1 \%)$ had received prior pelvic radiation. Eighteen patients $(78.3 \%)$ had received one prior cytotoxic regimen, and five patients $(21.7 \%)$ had received two prior cytotoxic regimens. In over $95 \%$ of patients, the prior therapy had been doxorubicin-based and/or gemcitabine plus a taxane (Table 1).

\section{Response to treatment and survival}

Confirmed partial response was observed in two of 23 patients $(8.7 \%, 90 \%$ marginally twosided, binomial confidence interval 1.6-24.9\%). There were no complete responses. Seven patients $(30.4 \%)$ had stable disease while $14(60.9 \%)$ had disease progression or died within two months of study entry. Only five patients received four or more cycles of therapy. The median number of cycles per patient was 1 (range 1-14).

Four of 23 patients (17.4\%, 90\% two-sided, binomial confidence interval 6.2\%-35.5\%) survived progression-free for at least six months from study entry. Based on the sample size attained in the first stage of accrual, at least four patients with tumor responses or at least five patients who survived progression-free for six months was required before the study could open accrual to a second stage. Therefore, the study was closed. The median PFS was 1.54 
months (95\% confidence interval 1.28-3.48 months). The median OS was 15.1 months (Figure 2).

\section{Adverse events}

A summary of treatment related adverse events (defined as having an attribution to the regimen of at least possible) for the worse grade experienced by the patients by organ (or organ system) is provided in Table 2. Myelosuppression was observed with the following frequencies: grade 3 neutropenia (17.3\%), grade 3 thrombocytopenia (13\%), and grade 3 anemia (17.3\%), and grade 3-4 lymphopenia (8.6\%). Constitutional symptoms were common with grade 3 fatigue in six patients (26\%) and grade 4 fatigue in one (4.3\%). Grade 3 weight loss was seen in one $(4.3 \%)$ patient. One patient $(4.3 \%)$ had grade 2 fever. Dermatologic toxicities (skin rash, handfoot syndrome, nail changes), grade 2, were observed in 3 patients (13\%) and were grade 3 in one patient $(4.3 \%)$. Gastrointestinal toxicities included grade 3 nausea $(8.7 \%)$, grade 3 vomiting $(8.7 \%)$, grade 3 diarrhea (13\%), grade 3 dehydration $(8.7 \%)$, and grade 3 anorexia $(4.3 \%)$. Hypertension grade 2 (defined as blood pressure $>150 / 100$ or a symptomatic increase in diastolic pressure of $>20 \mathrm{mmHg}$ from baseline) developed in 9 of 23 patients $(39 \%)$ and one patient had grade 3 hypertension (4.3\%). One patient had a grade 2 decrease in left ventricular ejection fraction. One patient had grade 3 thrombosis (4.3\%) during study treatment, and died. The GOG Data Safety and Monitoring Board determined that the cause of death was both study treatment and disease.

\section{DISCUSSION}

Anti-angiogenic agents have shown activity in some cytotoxic agent-refractory solid tumors such as renal cell cancer and gastrointestinal stromal tumors (GIST). ${ }^{30,31}$ Since it was considered that an anti-angiogenic agent may achieve, at best, disease stabilization rather than objective response, this phase II trial was designed with a dual endpoint such that either objective response or surviving progression-free at six months would be considered a "success." Specifically, the study was designed to determine whether daily oral sunitinib (four weeks on and two weeks off) could achieve a sufficient number of objective responses or maintain enough patients progression-free for at least six months to consider the regimen for investigation in a fully powered randomized phase III study. Sunitinib treatment failed to meet either of these criteria and can be considered inactive as second or third-line therapy for advanced uterine LMS.

These sunitinib results can be compared with other agents that have been tested in the secondline metastatic LMS setting. Gemcitabine as bolus infusion, single-agent therapy for patients with $0-1$ prior cytotoxic regimens achieved response in $20 \%$ of patients. ${ }^{8} \mathrm{~A}$ fixed-dose rate gemcitabine plus docetaxel regimen achieved objective responses in $27 \%$ of patients as secondline therapy. ${ }^{6}$ Trabectedin achieved objective responses in $8 \%$ of soft tissue sarcoma patients as second-line therapy. ${ }^{10}$ Among multi-kinase inhibitor anti-angiogenic agents, in a phase II study of sorafenib for patients with soft tissue sarcoma who had had 0-1 prior therapies, sorafenib achieved one response in 37 patients $(3 \%)$ with LMS. ${ }^{32}$ Sunitinib was studied in a phase II study for patients with non-GIST sarcomas. Among 20 patients in the histologic cohort that was enriched for sarcoma types that had been reported to respond to tyrosine kinase inhibitors (including 5 patients with LMS), there were no objective responses. ${ }^{33}$ A separate phase II study of sunitinib enrolled 36 patients with non-GIST sarcoma (12 with LMS) with one objective response seen. ${ }^{34}$

The side effect profile for sunitinib in the uterine LMS population is of interest. Myelosuppression, hypertension, and fatigue are common. Although gastrointestinal toxicities consisting of nausea, vomiting and diarrhea were fairly common, there were no intestinal perforations. However, given the small number of patients treated and the short duration of 
treatment exposure due to disease progression, it is unlikely that we would detect this relatively rare but serious toxicity in this trial.

The PFS rate at three months and six months has been proposed as an end-point for phase II trials in soft tissue sarcoma. It is suggested that first-line treatments should achieve progressionfree rates of $\geq 30-56 \%$ at six months, and second-line agents should achieve three-month progression-free rates of $\geq 40 \% .{ }^{35}$ By these criteria, sunitinib would be considered inactive with fewer than $40 \%$ of patients progression-free at three months, and only $17.4 \%$ of patients progression-free at six months. In contrast, $52 \%$ of uterine LMS patients treated with fixed dose-rate gemcitabine plus docetaxel as second-line therapy were progression-free at six months, ${ }^{6}$ and $18 \%$ of patients treated with single agent gemcitabine on a separate phase II trial were progression-free at six months ${ }^{8}$. The Gynecologic Oncology Group has tested a number of other agents in phase II trials for patients with uterine leiomyosarcoma who had received one prior treatment regimen. The percent of patients surviving progression-free at six months was fewer than $15 \%$ for the following agents: paclitaxel $^{18}$, trimetrexate $^{21}$, thalidomide ${ }^{20}$.

Sunitinib fails to achieve objective response or sustained disease stabilization as second or third-line therapy among patients with advanced uterine leiomyosarcoma.

\section{Supplementary Material}

Refer to Web version on PubMed Central for supplementary material.

\section{Acknowledgments}

The authors wish to thank Sandra Dascomb for her support with data acquisition and reconciliation as well as Kim Blaser for her help with publication and Debra Heller for histologic review.

This study was supported by National Cancer Institute grants to the Gynecologic Oncology Group Administrative Office (CA 27469), and the Gynecologic Oncology Group Statistical and Data Center (CA 37517). The following Gynecologic Oncology Group member institutions participated in this study: University of North Carolina at Chapel Hill School of Medicine, Memorial Sloan-Kettering Cancer Center, The University of Texas MD Anderson Cancer Center, Fox Chase Cancer Center, University of Oklahoma, Case Western Reserve University, University of Wisconsin, Women and Infants-Brown University, Central Connecticut Hospital, and Community Clinical Oncology Program.

\section{REFERENCES}

1. Major FJ, Blessing JA, Silverberg SG, et al. Prognostic factors in early-stage uterine sarcoma: A Gynecologic Oncology Group Study. Cancer 1993;71:1702-1709. [PubMed: 8381710]

2. Levenback C, Rubin SC, McCormack PM, et al. Resection of pulmonary metastases from uterine sarcomas. Gynecol Oncol 1992;45:202-205. [PubMed: 1592288]

3. Sutton G, Blessing JA, Malfetano JH. Ifosfamide and doxorubicin in the treatment of advanced leiomyosarcomas of the uterus: a Gynecologic Oncology Group study. Gynecol Oncol 1996;62:226229. [PubMed: 8751554]

4. Hensley ML, Maki R, Venkatraman E, et al. Gemcitabine and Docetaxel in Patients with Unresectable Leiomyosarcoma: Results of a Phase II Trial. J Clin Oncol 2002;20:2824-2831. [PubMed: 12065559]

5. Hensley ML, Blessing JA, Mannel R, et al. Fixed-dose rate gemcitabine plus docetaxel as first-line therapy for metastatic uterine leiomyosarcoma: a Gynecologic Oncology Group phase II trial. Gynecol Oncol 2008;109:329-334. [PubMed: 18534250]

6. Hensley ML, Blessing JA, DeGeest K, et al. Fixed-dose rate gemcitabine plus docetaxel as secondline therapy for metastatic uterine leiomyosarcoma: a Gynecologic Oncology Group phase II study. Gynecol Oncol 2008;109:323-328. [PubMed: 18394689]

7. Sutton GP, Blessing JA, Barrett FJ, et al. Phase II trial of ifosfamide and mesna in leiomyosarcoma of the uterus: a Gynecologic Oncology Group study. Am J Obstet Gynecol 1992;166:556-559. [PubMed: 1536229] 
8. Look KY, Sandler A, Blessing JA, et al. Phase II trial of gemcitabine as second-line chemotherapy of uterine leiomyosarcoma: a Gynecologic Oncology Group (GOG) study. Gynecol Oncol 2004;92:644647. [PubMed: 14766260]

9. Garcia-Carbonero R, Supko JG, Maki RG, et al. Ecteinascidin- 743 (ET-743) for chemotherapy-naive patients with advanced soft tissue sarcomas: multicenter phase II and pharmacokinetic study. J Clin Oncol 2004;23:5484-5492. [PubMed: 16110008]

10. Delaloge S, Yovine A, Taamma A, et al. Ecteinasidin-743: a marine-derived compound in advanced, pre-treated sarcoma patients—preliminary evidence of activity. J Clin Oncol 2001;19:1248-1255. [PubMed: 11230465]

11. Thigpen JT, Blessing JA, Beecham J, et al. Phase II trial of cisplatin as first-line chemotherapy in patients with advanced or recurrent uterine sarcomas: a Gynecologic Oncology Group study. J Clin Oncol 1991;9:1962-1966. [PubMed: 1941054]

12. Muss HB, Bundy BN, Adcock L, et al. Mitoxantrone in the treatment of advanced uterine sarcoma: A phase II trial of the Gynecologic Oncology Group. Am J Clin Oncol 1990;13:32-34. [PubMed: 2154921]

13. Asbury R, Blessing JA, Buller R, et al. Amonafide in patients with leiomyosarcoma of the uterus: a phase II Gynecologic Oncology Group study. Am J Clin Oncol 1998;21:145-146. [PubMed: 9537200]

14. Rose PG, Blessing JA, Soper JT, et al. Prolonged oral etoposide in recurrent or advanced leiomyosarcoma of the uterus: a Gynecologic Oncology Group study. Gynecol Oncol 1998;70:267271. [PubMed: 9740703]

15. Slayton RE, Blessing JA, Look K, et al. A Phase II clinical trial of diazoquone (AZQ) in the treatment of patients with recurrent leiomyosarcoma of the uterus: a Gynecologic Oncology Group study. Invest New Drugs 1991;9:207-208. [PubMed: 1874603]

16. Slayton R, Blessing J, Angel C, et al. Phase II trial of etoposide in the management of advanced or recurrent leiomyosarcoma of the uterus: a Gynecologic Oncology Group study. Cancer Treat Rep 1987;71:1303-1304. [PubMed: 3690545]

17. Miller DS, Blessing JA, Kilgore LC, et al. Phase II trial of topotecan in patients with advanced, persistent, or recurrent uterine leiomyosarcomas: a Gynecologic Oncology Group Study. Am J Clin Oncol 2000;23:355-357. [PubMed: 10955863]

18. Gallup DG, Blessing JA, Anderson W, et al. Evaluation of paclitaxel in previously treated leiomyosarcoma of the uterus: a Gynecologic Oncology Group study. Gynecol Oncol 2003;89:4851. [PubMed: 12694653]

19. Sutton G, Blessing JA, Ball H. Phase II trial of paclitaxel in leiomyosarcoma of the uterus: a Gynecologic Oncology Group study. Gynecol Oncol 1999;74:346-349. [PubMed: 10479491]

20. McMeekin DS, Sill M, Benbrook D, et al. A Phase II trial of thalidomide in patients with refractory endometrial cancer and correlation with angiogenesis biomarkers: a Gynecologic Oncology Group Study. Gynecol Oncol 2007;105:508-516. [PubMed: 17306350]

21. Smith HO, Blessing JA, Vaccarello L. Trimetrexate in the treatment of recurrent or advanced leiomyosarcoma of the uterus: a phase II study of the Gynecologic Oncology Group. Gynecol Oncol 2002;84:140-144. [PubMed: 11748990]

22. Olson T, Mohanraj D, Carson LF, et al. Vascular permeability factor gene expression in normal and neoplastic human ovaries. Cancer Res 1994;54:276-280. [PubMed: 8261452]

23. Guidi AJ, Abu-Jawdeh G, Berse G, et al. Vascular permeability factor (vascular endothelial growth factor) expression and angiogenesis in cervical neoplasia. J Natl Cancer Inst 1995;87:1237-1245. [PubMed: 7563170]

24. Guidi AJ, Abu-Jawdeh G, Tognazzxi K, et al. Expression of vascular permeability factor (vascular endothelial growth factor) and its receptors in endometrial carcinoma. Cancer 1996;78:454-460. [PubMed: 8697391]

25. Chen CA, Cheng WF, Lee CN, et al. Serum vascular endothelial growth factor in epithelial ovarian neoplasms: correlation with patient survival. Gynecol Oncol 1999;74:235-240. [PubMed: 10419737]

26. Holash J, Davis S, Papadopoulos N, et al. VEGF-Trap: A VEGF blocker with potent antitumor effects. Proc Natl Acad Sci USA 2002;99:11393-11398. [PubMed: 12177445] 
27. Dupont J, Rothenberg ML, Spriggs DR, et al. Safety and pharmacokinetics of intravenous VEGFTrap in a phase I clinical trial of patients with advanced solid tumors. Proc Amer Soc Clin Oncol 2005;23abstract 3029

28. Investigator's Brochure SU011248 (December 2005). New York: Pfizer, Inc. and Affiliates, New York;

29. Sill, MW.; Yothers, G. Technical Report 2006-08. Department of Biostatistics, University at Buffalo; A method for utilizing bivariate efficacy outcome measures to screen agents for activity in 2-stage phase II clinical trials.

30. Motzer RJ, Michaelson MD, Redman BG, et al. Activity of SU11248, a multitargeted inhibitor of vascular endothelial growth factor receptor and platelet-derived growth factor receptor, in patients with metastatic renal cell carcinoma. J Clin Oncol 2006;24:16-24. [PubMed: 16330672]

31. Demetri GD, van Oosterom AT, Garrett CR, et al. Efficacy and safety of sunitinib in patients with advanced gastrointestinal stromal tumour after failure of imatinib: a randomised controlled trial. Lancet 2006;368:1329-1338. [PubMed: 17046465]

32. Maki RG, D'Adamo DR, Keohan ML, et al. A phase II study of sorafenib in patients with metastatic/ recurrent sarcomas. J Clin Oncol. 2009In press

33. Keohan ML, Morgan JA, D'Adamo DR, et al. Continuous daily dosing (CDD) of sunitinib (SU) in patients with metastatic soft tissue sarcomas (STS) other than GIST: Results of a phase II trial. J Clin Oncol 2008;26(May 20 suppl; abstr 10533)

34. Vigil CE, Chiappori AA, Williams CA, et al. Phase II study of sunitinib malate (SM) in subjects with metastatic and/or surgically unresectable non-GIST soft tissue sarcomas. J Clin Oncol 2008;26(May 20 suppl; abstr 10535)

35. Van Glabbeke M, Verweij J, Judson I, et al. Progression-free rate as the principal end-point for phase II trials in soft tissue sarcoma. Eur J Cancer 2002;38:543-549. [PubMed: 11872347] 


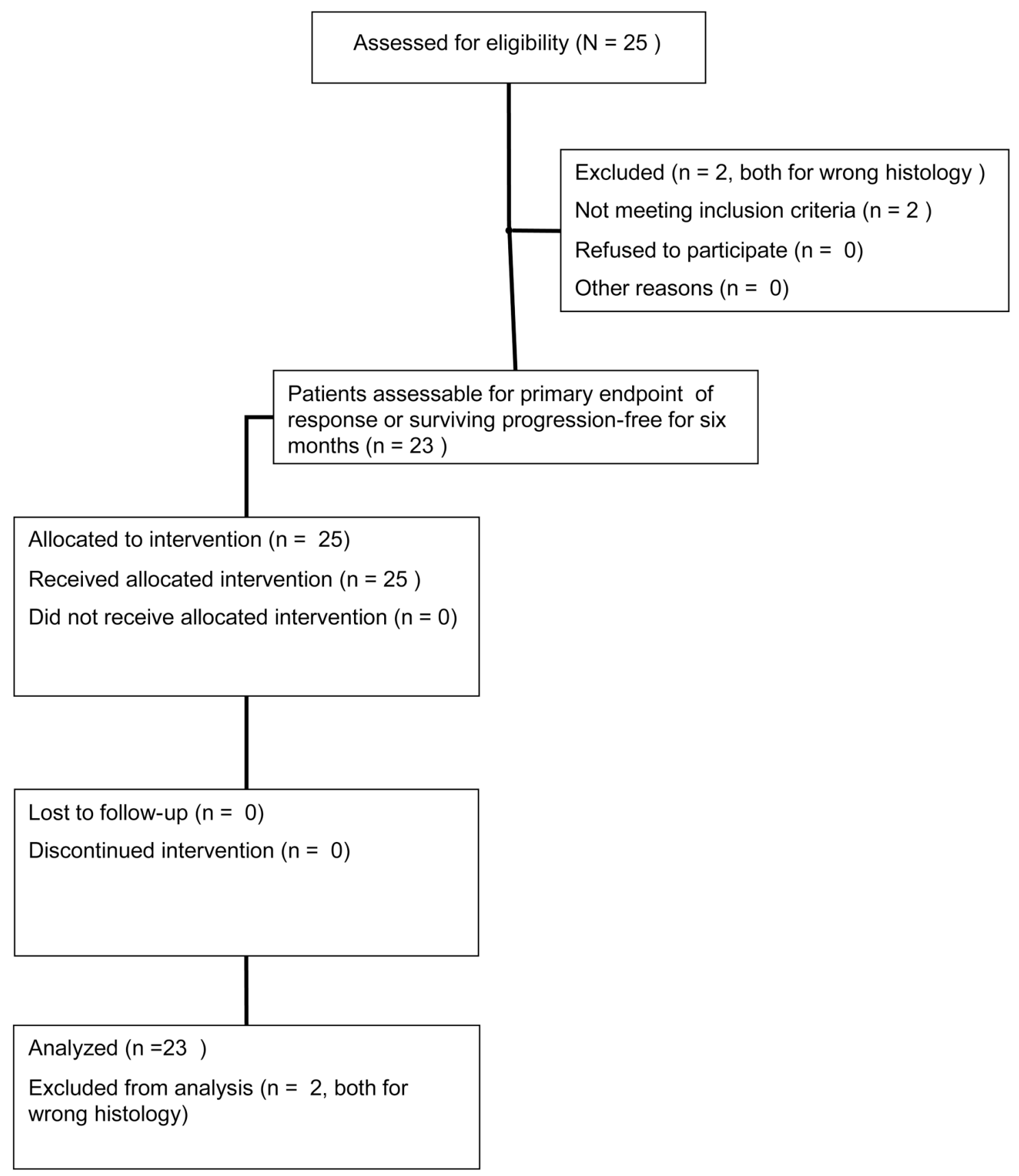

Figure 1.

CONSORT diagram of patient enrollment on phase II trial of sunitinib for the treatment of recurrent or persistent uterine leiomyosarcoma. 


\section{Overall and Progression-Free Survival}

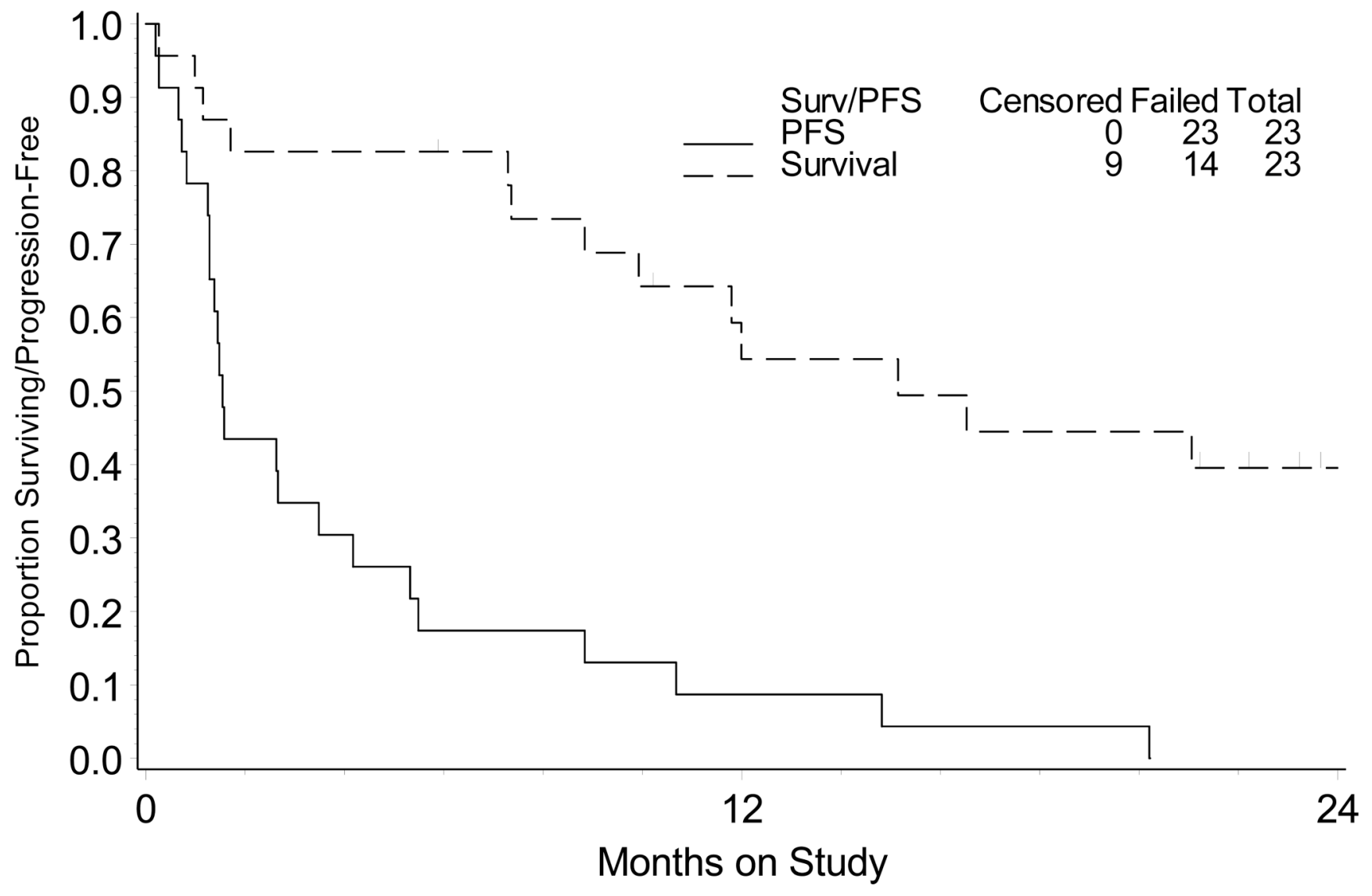

Figure 2.

Progression-free survival and overall survival among patients with advanced uterine leiomyosarcoma treated with sunitinib $(n=23)$. Median PFS is 1.54 months; $(95 \%$ confidence interval 1.28, 3.48). Median overall survival is 15.1 months.

PFS: progression-free survival 
Table 1

Patient characteristics $(n=23)$

\begin{tabular}{|c|c|c|}
\hline Characteristic & $\begin{array}{r}\text { Number of } \\
\text { Cases }\end{array}$ & $\%$ \\
\hline \multicolumn{3}{|l|}{ Age, years } \\
\hline $30-39$ & 3 & 13 \\
\hline $40-49$ & 3 & 13 \\
\hline $50-59$ & 10 & 43.5 \\
\hline $60-69$ & 5 & 21.7 \\
\hline $70-79$ & 2 & 8.7 \\
\hline \multicolumn{3}{|l|}{ Race } \\
\hline African American & 4 & 17.4 \\
\hline American Indian & 1 & 4.3 \\
\hline White & 18 & 78.3 \\
\hline \multicolumn{3}{|l|}{ Performance Status } \\
\hline 0 & 13 & 46.5 \\
\hline 1 & 9 & 39.1 \\
\hline 2 & 1 & 4.3 \\
\hline \multicolumn{3}{|l|}{ Cell Type } \\
\hline Leiomyosarcoma & 23 & 100 \\
\hline \multicolumn{3}{|l|}{ Prior Chemotherapy } \\
\hline One regimen & 18 & 78.3 \\
\hline Two regimens & 5 & 21.7 \\
\hline \multicolumn{3}{|l|}{ Prior Radiation } \\
\hline No & 14 & 60.9 \\
\hline Yes & 9 & 39.1 \\
\hline
\end{tabular}




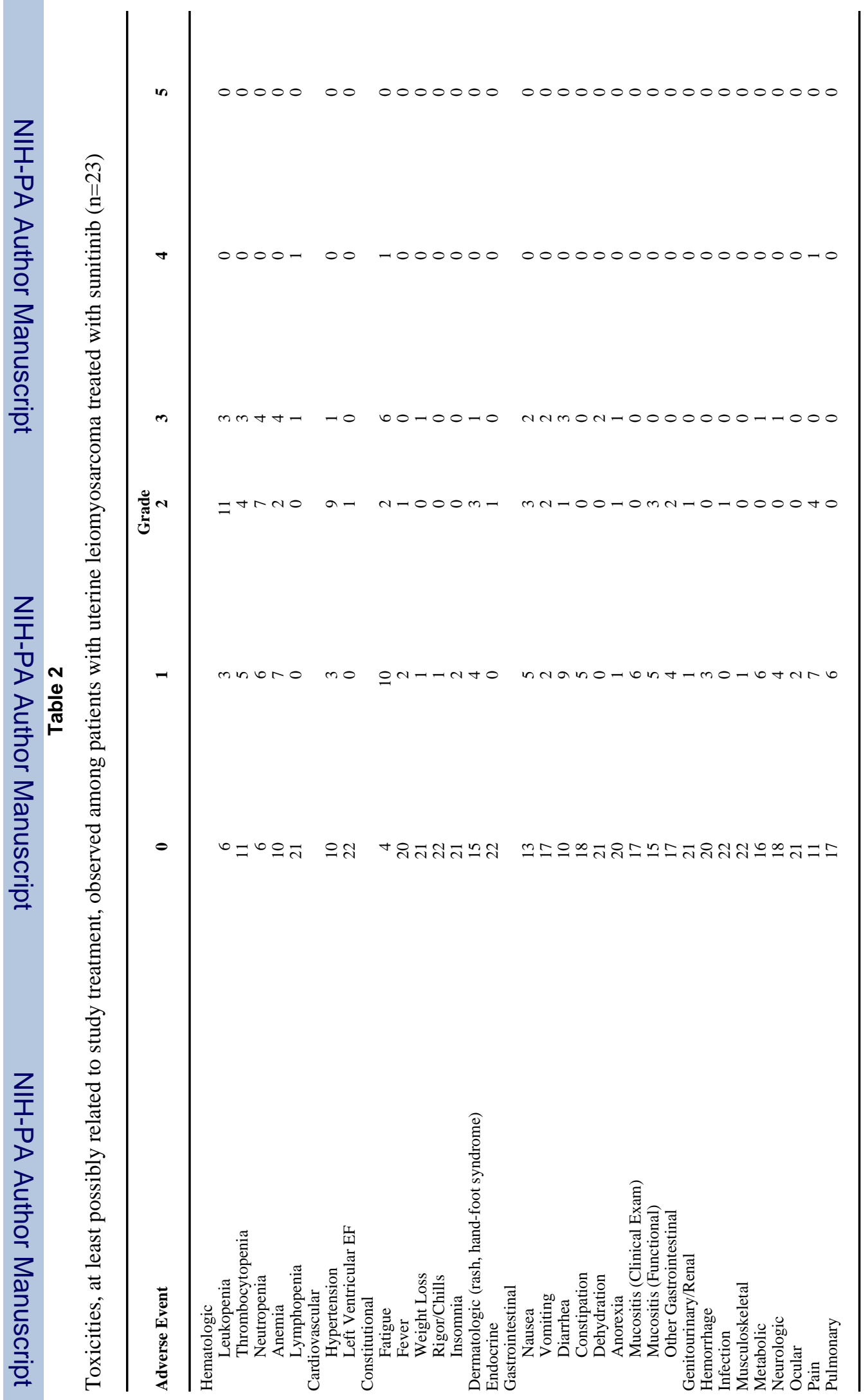

Gynecol Oncol. Author manuscript; available in PMC 2010 December 1. 